\title{
Structural and magnetic properties of Co-Mn-Sb thin films
}

\author{
Markus Meinert, ${ }^{1}$, a) Jan-Michael Schmalhorst, ${ }^{1}$ b) Daniel Ebke, ${ }^{1}$ Ning-Ning Liu, ${ }^{1}$ Andy Thomas, ${ }^{1}$ Günter Reiss, ${ }^{1}$ \\ Jaroslaw Kanak, ${ }^{2}$ Tomasz Stobiecki, ${ }^{2}$ and Elke Arenholz ${ }^{3}$ \\ 1) Thin Films and Physics of Nanostructures, Department of Physics, Bielefeld University, 33501 Bielefeld, \\ Germany \\ ${ }^{2)}$ Department of Electronics, University of Science and Technology, 30059 Krakow, \\ Poland \\ ${ }^{3)}$ Lawrence Berkeley National Laboratory, Berkeley, CA 94720, USA
}

(Dated: 28 June 2018)

Thin Co-Mn-Sb films of different compositions were investigated and utilized as electrodes in alumina based magnetic tunnel junctions with CoFe counter electrode. The preparation conditions were optimized with respect to magnetic and structural properties. The Co-Mn-Sb/Al-O interface was analyzed by X-ray absorption spectroscopy and magnetic circular dichroism with particular focus on the element-specific magnetic moments. Co-Mn-Sb crystallizes in different complex cubic structures depending on its composition. The magnetic moments of $\mathrm{Co}$ and $\mathrm{Mn}$ are ferromagnetically coupled in all cases. A tunnel magneto resistance ratio of up to $24 \%$ at $13 \mathrm{~K}$ was found and indicates that Co-Mn-Sb is not a ferromagnetic half-metal. These results are compared to recent works on the structure and predictions of the electronic properties.

PACS numbers: 75.70.-i, 78.70.Dm, 85.75.-d

\section{INTRODUCTION}

Half-metallic materials are characterized by a gap in the density of states for minority (or majority) electrons at the Fermi energy, giving a spin polarization of $P=100 \%$. Therefore these materials are of great interest for spintronic devices such as magnetic sensors, nonvolatile memories $\frac{1}{1}$ or programmable logic devices ${ }^{2,3}$. Taking Jullière's simple model ${ }^{4}$ of the tunnel magnetoresistance, an infinite TMR ratio is expected if two halfmetals are used as ferromagnetic electrodes in magnetic tunnel junctions (MTJs).

Several half and full Heusler compounds have been predicted to be half metals ${ }^{-\frac{9}{-9}}$ and implemented in MTJs. The largest spin polarizations in Al-O based junctions were reported for the full Heusler compounds $\mathrm{Co}_{2} \mathrm{Cr}_{1-x} \mathrm{Fe}_{x} \mathrm{Al}^{10}$ and $\mathrm{Co}_{2} \mathrm{YZ}(\mathrm{Y}=\mathrm{Mn}$ or $\mathrm{Fe} ; \mathrm{Z}=\mathrm{Si}$ or $\mathrm{Al})^{11-16}$ after Tanaka et al. ${ }^{17}$ started investigating $\mathrm{NiMnSb}$ in 1999. $\mathrm{Co}_{2} \mathrm{MnSi}$ electrodes combined with $\mathrm{MgO}$ barriers yield even higher TMR ratios ${ }^{18}$. However, the highest TMR ratios are currently found in Co-Fe$\mathrm{B} / \mathrm{MgO} / \mathrm{Co}-\mathrm{Fe}-\mathrm{B}$ junctions ${ }^{19}$.

Ideally, a full Heusler compound $\mathrm{X}_{2} \mathrm{YZ}$ crystallizes in $L 2_{1}$ structure whereas a half Heusler compound XYZ crystallizes in $C 1_{b}$ structure. Real Heusler compounds often show a certain degree of atomic disorder and thus deviate from the ideal structures. Generally, this has a strong influence on the electronic properties of the films such as the spin polarization.

Here, Co-Mn-Sb is implemented as an electrode in magnetic tunnel junctions. CoMnSb was thought to be a half Heusler compound and the structural, magnetic

\footnotetext{
a) Electronic mail: meinert@physik.uni-bielefeld.de

b) Electronic mail: jschmalh@physik.uni-bielefeld.de
}

and electronic properties of bulk CoMnSb were controversially discussed in the literature. Galanakis ${ }^{20}$ assumed the $C 1_{b}$ structure for CoMnSb and calculated its band structure. The total spin magnetic moment was almost $3 \mu_{B}$ per formula unit (f.u.), while the $\mathrm{Mn}\left(3.18 \mu_{B}\right)$ and the Co $\left(-0.13 \mu_{B}\right)$ spin moments were antiferromagnetically aligned. Presuming a typical lattice constant of $5.9 \AA$ this corresponds to a low temperature saturation magnetization of $540 \mathrm{kA} / \mathrm{m}$. Half metallicity with a large spin-down gap of about $1 \mathrm{eV}$ was proposed for this structure. However, a total magnetic moment of $3 \mu_{B}$ per f.u. is in contradiction to an experimentally observed value of about $4 \mu_{B}$ per f.u. ${ }^{21}$. As discussed by Tobola et al. ${ }^{22}$ this larger moment can be explained by a more complex structure (space group $F d \overline{3} m$ ), which was originally proposed by Senateur et al. ${ }^{23}$ in 1972. A narrow spin-down gap is found for this structure, but the half-metallic character is preserved. The calculated mean $\mathrm{Mn}$ and Co magnetic moments are $3.51 \mu_{B}$ and $0.48 \mu_{B}$, respectively, and are expected to couple ferromagnetically.

Kaczmarska et al ${ }^{24}$ could also reproduce a magnetic moment of nearly $4 \mu_{B}$ per f.u. by their calculations, where a 'disordered' $L 2_{1}$ structure was assumed. The two X sublattices were occupied by $50 \%$ Co and $50 \%$ vacant sites and the $\mathrm{Y}$ by $\mathrm{Mn}$ and $\mathrm{Z}$ by Sb. The ferromagnetically coupled Mn and Co magnetic moments were calculated to be $3.60 \mu_{B}$ and $0.30 \mu_{B}$ and half-metallicity disappeared.

Recently, Ksenofontov et al.$^{25}$ revised the structure of CoMnSb bulk samples using XRD, NMR and Mössbauer spectroscopy. They assigned it to space group $F m \overline{3} m$ and presented the $\mathrm{CoMnSb}$ structure as an alternation of $\mathrm{Co}_{2} \mathrm{MnSb}$ and $\mathrm{MnSb}$ structural units with a calculated Co moment of $0.55 \mu_{B}$ and a mean Mn moment of $3.48 \mu_{B}$. Taking the experimental lattice parameter of $11.73 \AA$ into account this corresponds to a magnetization of $740 \mathrm{kA} / \mathrm{m}$. CoMnSb crystallized in this structure is 
predicted to be not half-metallic.

In this paper, we present investigations of Co-Mn$\mathrm{Sb}$ thin films of different compositions implemented in MTJs with Al-O barrier and Co-Fe counter electrode. In Sec. III we analyze the bulk properties of the films, namely magnetization, interdiffusion with the buffer, element specific magnetic moments and crystal structure. Optimized growth conditions of the films were obtained with respect to these quantities. In Sec. IV we discuss the properties of the Co-Mn-Sb / Al-O interface and the tunnel properties of the corresponding magnetic tunnel junctions.

\section{EXPERIMENTAL}

The samples were deposited at room temperature by DC-magnetron sputtering in a sputter tool with a base pressure of $2 \cdot 10^{-7}$ mbar. Thermally oxidized $\mathrm{Si}(100)$ wafers and $200 \mathrm{~nm}$ thick $\mathrm{Si}_{3} \mathrm{~N}_{4}$ membranes with a window size of $3 \mathrm{~mm} \times 3 \mathrm{~mm}$ were used as substrates. First, a vanadium buffer was deposited, then, the Co-Mn-Sb films were prepared from three different targets. The nominal

\begin{tabular}{cccc} 
target & $\mathrm{Co}$ & $\mathrm{Mn}$ & $\mathrm{Sb}$ \\
\hline I & 29.0 & 32.1 & 38.9 \\
II & 33.3 & 33.3 & 33.3 \\
III & 35.1 & 29.4 & 35.5
\end{tabular}

\begin{tabular}{cccc} 
film & $\mathrm{Co}$ & $\mathrm{Mn}$ & $\mathrm{Sb}$ \\
\hline I & 32.4 & 33.7 & 33.8 \\
II & 37.7 & 34.1 & 28.2 \\
III & 43.2 & 32.6 & 24.2
\end{tabular}

TABLE I. Nominal target and the resulting film compositions. Film II was analyzed by inductively coupled plasma optical emission spectroscopy (ICP-OES) and energy dispersive x-ray spectroscopy (EDX). Identically prepared films from targets I and III were investigated by EDX and corrected using the ICP-OES values of film II. The observed edge jump heights in the x-ray absorption spectra of Co and Mn agree with these concentrations.

target and the resulting film compositions are listed in Tab. I. The films were capped by a thin Al layer to prevent unintentional oxidation of the Co-Mn-Sb or - in combination with plasma oxidation - to form a tunnel barrier. The layer sequences and notation of the samples discussed in this paper are given in Tab. III

\section{THIN CO-MN-SB FILMS ON V BUFFERS: BULK PROPERTIES}

\section{A. Magnetization studies: Alternating gradient field magnetometry (AGM)}

The bulk magnetization of the samples was measured at room temperature in an AGM by Princeton Measurements, Inc. The saturation value $M_{S}$ of sample Half $\mathrm{B}^{I}$ in dependence of the annealing temperature $T_{A}$ is shown in Figure 17. The largest RT magnetization of $508 \mathrm{kA} / \mathrm{m}$ is found after annealing at $400^{\circ} \mathrm{C}$. The magnetization at low
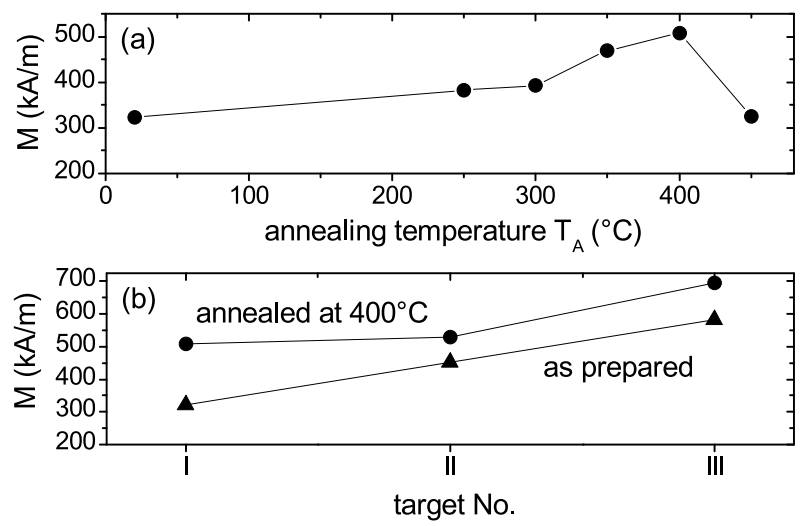

FIG. 1. Bulk saturation magnetization $M_{S}$ at room temperature: (a) Annealing temperature $T_{A}$ dependence of $M_{S}$ for sample Half $\mathrm{B}^{I}$. (b) Influence of target composition on $M_{S}$ for samples of type Half B.

temperature is significantly higher than at RT because of the relatively low Curie temperature of about $480 \mathrm{~K}^{21,25}$. We observed a $15 \%$ reduction of the $\mathrm{Mn}$ spin moment of sample Half $\mathrm{A}^{I}$ between $T=15 \mathrm{~K}$ and RT (cp. Sec. III C). Therefore we estimate $M_{S}(15 \mathrm{~K})=600 \mathrm{kA} / \mathrm{m}$. A similar increase of the magnetization with increasing annealing temperature is found for sample Half $\mathrm{B}^{I I, I I I}$ (see Fig. 1b). Furthermore, the bulk magnetization increases with increasing Co concentration of the films, which is highest for target III.

\section{B. Inter-diffusion of Co-Mn-Sb and V: Auger depth profiling}

The decrease of the magnetic moment above the optimal annealing temperature is caused by inter-diffusion of the Co-Mn-Sb layer with the V buffer. This is identified by Auger depth profiling 26,27 of sample Half $\mathrm{B}^{I}$ samples in the as prepared state and after annealing at $350^{\circ} \mathrm{C}$ and $450^{\circ} \mathrm{C}$ (Figure 2). The different layers are well separated in the as prepared state. The apparent maximum of the Mn intensity just below the barrier is caused by preferential sputtering of Manganese. It is a very common artifact of sputter depth profiling 27 and was also found for Antimony. After annealing at $350^{\circ} \mathrm{C}$ the depth profile is slightly changed, a small fraction of Vanadium could be detected in the lower part of the Co-Mn-Sb layer (see arrow in Figure 2). This inter-diffusion intensifies with increasing annealing temperature. Therefore the annealing temperature for samples Half A, Half $\mathrm{C}^{I I}$ and Full ${ }^{I I}$ was generally set to $350^{\circ} \mathrm{C}$ to minimize diffusion.

In addition Auger depth profiles were taken for the Co rich samples Half $\mathrm{B}^{I I I}$. After annealing at $450^{\circ} \mathrm{C}$ the $\mathrm{V}$ concentration in the depth region of the $\mathrm{Co}-\mathrm{Mn}-\mathrm{Sb}$ layer was a factor of two smaller for sample Half $\mathrm{B}^{I I I}$ compared to sample Half $\mathrm{B}^{I}$, i.e. the inter-diffusion at the Co$\mathrm{Mn}-\mathrm{Sb} / \mathrm{V}$ interface is considerably smaller for the off- 
sample layer sequence

\begin{tabular}{|c|c|c|c|}
\hline Half $\mathrm{A}^{I, I I, I I I}$ & $\mathrm{Si}_{3} \mathrm{~N}_{4}$-membrane/ & V $20(\mathrm{~nm}) / \mathrm{Co}-\mathrm{Mn}-\mathrm{Sb} 40 /$ & Al 1.6 \\
\hline Half $\mathrm{B}^{I, I I, I I I}$ & Si-wafer $/ \mathrm{SiO}_{2}$ 50/ & Co-Mn-Sb 100/ & Al 1.6 \\
\hline Half $\mathrm{C}^{I I}$ & Si-wafer $/ \mathrm{SiO}_{2}$ 50/ & Co-Mn-Sb 100/ & $\mathrm{Al} 0-3 \mathrm{~nm}$ wedge $+150 \mathrm{~s}$ plasma oxidation \\
\hline Full $^{I I}$ & $\begin{array}{l}\mathrm{Si} \text {-wafer } / \mathrm{SiO}_{2} 50 / \\
+60 \min 350^{\circ} \mathrm{C} \text { in-situ }\end{array}$ & $\begin{array}{l}\text { Co-Mn-Sb } 100 / \\
+50 \text { s plasma }\end{array}$ & $\begin{array}{l}\text { Al } 0-3 \mathrm{~nm} \text { wedge }+150 \text { s plasma oxidation } \\
\text { oxidation }+\mathrm{Co}_{70} \mathrm{Fe}_{30} 5 / \mathrm{Mn}_{83} \operatorname{Ir}_{17} 10 /+ \text { capping }\end{array}$ \\
\hline
\end{tabular}

TABLE II. Layer sequences of all samples. The sample series Half A and Half C were prepared for XAS and XMCD studies. Structural and magnetic bulk properties were determined on Half B samples. The transport properties of MTJs were measured on series Full. The roman superscript indicates the target number.

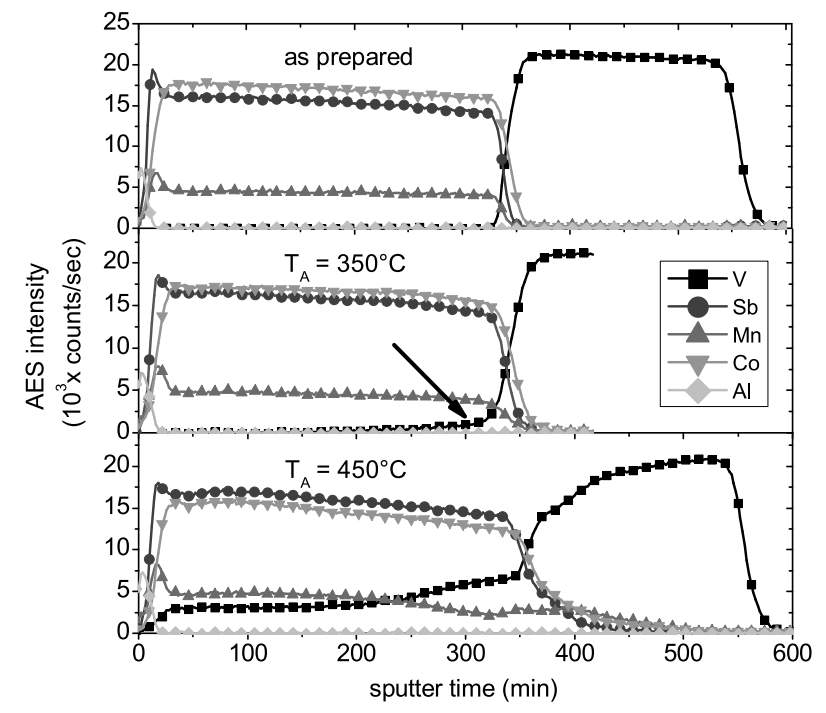

FIG. 2. Auger electron spectroscopy (AES) depth profiles of sample Half $\mathrm{B}^{I}$. V diffuses into Co-Mn-Sb at high annealing temperature. The depth resolution is not influenced by the annealing procedure ${ }^{28}$.

stoichiometric samples. This indicates that the number of vacancy sites via which bulk diffusion can easily occur in Co-Mn-Sb decreases with increasing Co concentration.

\section{Element-specific magnetic moments: $\mathrm{X}$-ray absorption spectroscopy (XAS) and magnetic circular dichroism (XMCD)}

Temperature dependent x-ray absorption spectroscopy (XAS) and x-ray magnetic circular dichroism (XMCD) was performed at BL 6.3.1 of the Advanced Light Source in Berkeley, CA. The element-specific magnetic bulk properties were investigated at the Co- and Mn- $L$ edges in bulk-sensitive transmission mode $\frac{29}{}$ on Half A samples in the as-prepared state and after annealing at $350^{\circ} \mathrm{C}$. The x-rays angle of incidence with respect to the sample surface was $\alpha=45^{\circ}$. The XMCD spectra were obtained by applying a magnetic field of $\pm 0.2 \mathrm{~T}$ along the $\mathrm{x}$ ray beam direction using elliptically polarized radiation with a polarization of $P_{h \nu}=+75 \% . \mu^{+}$and $\mu^{-}$denote the linear absorption coefficients for parallel and antiparallel orientation of photon spin and magnetic field. The transmitted $\mathrm{x}$-rays were detected by a photo-diode. In conventional sum rule analysis 30 the number of holes $N_{h}$ needs to be taken from band structure calculations to calculate element-specific spin and orbital magnetic moments. Here, we follow the approach by Stöhr et al. ${ }^{31}$ which bases on the assumption that the transition matrix elements connecting $2 p$ core and $3 d$ valence states are known and can be taken from other experiments. Details of our sum rule analysis procedure are given elsewhere ${ }^{32}$.

The XMCD spectra are shown in Fig. 3 a and b, the extracted magnetic moment ratios are summarized in Tab. [II]. The shape of the Mn XMCD asymmetry is nearly the same for all samples and very similar to $\mathrm{Mn}$ in the full Heusler compound $\mathrm{Co}_{2} \mathrm{MnSi} 33$. Accordingly, the $\mathrm{Mn}$ orbital to spin moment ratio does not depend on layer composition or post-annealing. The small Mn orbital moments are typically $-6 \%$ of the spin moments, i.e. both are aligned anti-parallel. Furthermore, the Mn magnetic moment of sample Half $\mathrm{A}^{I}$ was found to be reduced by $15 \%$ between $15 \mathrm{~K}$ and $\mathrm{RT}$, which is in fair agreement with the data by Otto et al. 21 , who observed a reduction of the saturation magnetization for bulk samples of $20 \%$ between $T=4 \mathrm{~K}$ and $\mathrm{RT}$ and a Curie temperature of $478 \mathrm{~K}$.

The Co spin moment is aligned in parallel to its small orbital moment and the target composition and the postannealing has a significant influence on the Co to Mn spin magnetic moments ratio which can be directly seen from the different sizes of the normalized Co XMCD signals in Fig. 3 b. Two clear trends are observed: First, the

\begin{tabular}{ccccc} 
target & & $m_{\text {orb }}^{M n} / m_{\text {spin }}^{M n}$ & $m_{\text {orb }}^{C o} / m_{\text {spin }}^{C o}$ & $m_{\text {spin }}^{C o} / m_{\text {spin }}^{M n}$ \\
\hline I & as prep. & $-5.9 \%$ & $7.6 \%$ & $27.4 \%$ \\
& $350^{\circ} \mathrm{C}$ & $-5.3 \%$ & $0.3 \%$ & $18.8 \%$ \\
\hline \multirow{2}{*}{ II } & as prep. & $-6.4 \%$ & $7.4 \%$ & $38.5 \%$ \\
& $350^{\circ} \mathrm{C}$ & $-7.3 \%$ & $5.3 \%$ & $29.6 \%$ \\
\hline \multirow{2}{*}{ III } & as prep. & $-5.5 \%$ & $7.0 \%$ & $45.5 \%$ \\
& $350^{\circ} \mathrm{C}$ & $-5.5 \%$ & $6.1 \%$ & $38.5 \%$ \\
\hline
\end{tabular}

TABLE III. Results of the sum rule analysis of the XMCD spectra shown in Fig. 3a and b for sample Half A. 

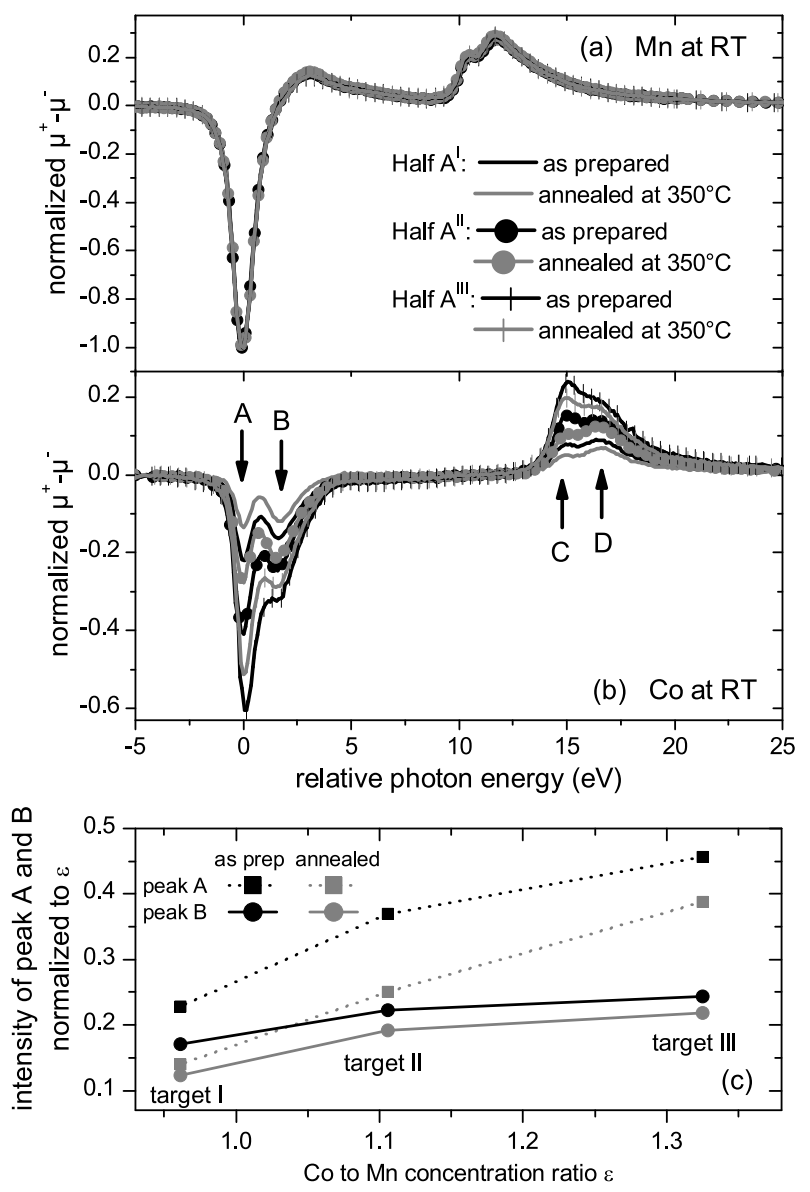

FIG. 3. XMCD spectra for sample Half $\mathrm{A}$ at the $\mathrm{Mn}-L_{2,3}$ (a) and Co- $L_{2,3}$ (b) edge. The spectra are normalized to the maximal XMCD signal at the Mn- $L_{3}$ edge for each sample. (c) Intensity of peak A and B normalized to the Co to Mn concentration ratio $\epsilon$.

normalized Co XMCD signal and, therefore, the relative contribution of each Co atom to the total magnetic moment of the samples is reduced by annealing for all samples of type Half A. Second, the relative contribution of the Co magnetic moments to the total magnetic moment of the samples increases with increasing Co concentration in the films. The smallest spin magnetic moment ratio $m_{\text {spin }}^{C o} / m_{\text {spin }}^{M n}$ of $18.8 \%$ is found for the annealed sample deposited from target I while the largest one $(45.5 \%)$ is found for the as prepared sample deposited from target III (see Tab. III). Similar results were found for annealed $\mathrm{Co}-\mathrm{Mn}-\mathrm{Sb}$ films grown on $\mathrm{Cu} / \mathrm{Ta} / \mathrm{V}$ buffers and are published elsewhere ${ }^{32}$.

Moreover, there is a remarkable double peak structure of the Co XMCD spectra at the $L_{2}$ and $L_{3}$ edge, which is significantly different from Co metal ${ }^{30}$ or $\mathrm{Co}_{2} \mathrm{MnSi}^{33}$ and leads to a strongly changed electronic structure of the unoccupied $3 d$ Co states compared to the references. This structure changes with different film composition and post-annealing. In addition to the decrease of the
Co to $\mathrm{Mn}$ spin moment ratio, which corresponds to generally reduced intensities of the peaks "A" and "B" (Fig. 35), the intensity of peak " $\mathrm{A}$ " is reduced further in comparison to "B" with decreasing Co concentration and by annealing. The same holds for the corresponding peaks "C" and "D" at the $L_{2}$ edge. The changed shape of the Co XMCD spectra could be caused by a different degree of location of the $3 \mathrm{~d}$ states 34 or hint to two nonequivalent Co positions in the Co-Mn-Sb crystal lattice. This is in accordance with the structural model of alternating $\mathrm{Co}_{2} \mathrm{MnSb}$ and $\mathrm{MnSb}$ structural units as discussed below. Moreover, because of the strong influence of the Co concentration on the spectral shape it is likely to assume, that the additional Co occupies the vacancy sites in the MnSb structural units.

The generally observed ferromagnetic coupling of the $\mathrm{Mn}$ and Co spin moment is important with respect to the different crystal structures discussed in the literature (see Sec. II). In the case of a $\mathrm{Cl}_{b}$ lattice an antiferromagnetic coupling of $\mathrm{Co}$ and $\mathrm{Mn}$ spin moments is expected $\left(m_{\text {spin }}^{C o} / m_{\text {spin }}^{M n}=-4.1 \%\right)$. For the more complex structures resulting in a very narrow or even vanishing spin-down gap, both moments are expected to be ferromagnetically coupled (Senateur's model: $m_{\text {spin }}^{C o} / m_{\text {spin }}^{M n}=$ $+13.7 \% \frac{22}{2}$; disordered $L 2_{1}$ structure: $m_{\text {spin }}^{C o} / m_{\text {spin }}^{M n}=$ $8.3 \%$; ; alternation of $\mathrm{Co}_{2} \mathrm{MnSb}$ and $\mathrm{MnSb}$ structural units: $\left.m_{\text {spin }}^{C o} / m_{\text {spin }}^{M n}=+15.8 \%{ }^{25}\right)$. Further, for CoMnSb crystallized in the $\mathrm{C}_{b}$ structure a parallel alignment of the spin and orbital moments is expected 35 . Obviously, our experimental data for the annealed Half $\mathrm{A}^{I}$ sample fits best together with the structural model proposed by Ksenofontov et al $\stackrel{25}{\underline{n}}$.

By comparing the element-specific results and the bulk magnetization discussed in Sec. III A one can conclude that the general increase of the magnetization with increasing Co content (see Fig. 1) results from the larger Co content and the larger contribution of each Co atom to the total magnetic moment. The general reduction of $m_{\text {spin }}^{C o} / m_{\text {spin }}^{M n}$ after annealing and the simultaneous increase of the bulk magnetization means, that the atomic order is improved by the post-annealing process: the magnetization of CoMnSb crystallized in one of the three complex structures suggested by Tobola et al $\stackrel{22}{\underline{2}}$, Kaczmarska et $a l^{24}$ and Ksenofontov et $a l^{25}$, , respectively, is dominated by the Mn spin moment, which is aligned in parallel to the Co spin moment. Thereby, the Co magnetic moment is considerably smaller in these compounds compared to pure Co metal $\left(m_{\text {spin }}^{C o} \approx 1.6 \mu_{B}{ }^{30}\right)$, where nearest-neighbor Co atoms couple ferromagnetically. From this one would expect an increase of the mean Co magnetic moment per atom in case of atomic disorder, e.g., when Co atoms occupy Mn lattice sites. On the other hand, nearest-neighbor $\mathrm{Mn}$ atoms tend to couple antiferromagnetically, accordingly a decrease of the mean Mn magnetic moment would be expected for a disordered compound. This behavior has been predicted by Picozzi et al. for defects in $L 2_{1}$ ordered $\mathrm{Co}_{2} \mathrm{MnSi}^{36}$. Accordingly, the results can be explained as an improvement of 


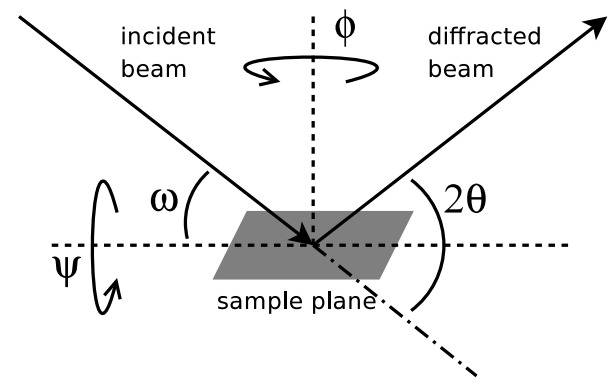

FIG. 4. The scattering geometry and the axes of the Philips X'Pert Pro MPD with open Eulerian cradle.

the atomic order by the post-annealing process. A similar behavior has been observed for full Heusler compound thin films and multilayers like $\mathrm{Co}_{2} \mathrm{MnSi}^{33}, \mathrm{Co}_{2} \mathrm{FeSi}$ and $\left\{\mathrm{Co}_{2} \mathrm{MnSi} / \mathrm{Co}_{2} \mathrm{FeSi}\right\} \times 10^{13,37}$.

\section{Crystal structure: X-ray diffraction (XRD)}

A Phillips X'Pert Pro MPD diffractometer equipped with an open Eulerian cradle and a copper anode was used for non-specular x-ray diffraction. Figure 4 displays the scattering geometry and the angle definitions. Crystallinity and atomic order of Co-Mn-Sb was studied and related to the magnetic and electronic properties.

Rocking curves and pole figures were taken to characterize the texture of the $\mathrm{V}$ buffer and the Co-Mn-Sb. Both materials grow (110) textured. The full width at half maximum (FWHM) of the Co-Mn-Sb $(440) /(220)$ rocking curves is $8^{\circ} \omega$ for sample Half $\mathrm{B}^{I}$ and $10^{\circ} \omega$ for sample Half $\mathrm{B}^{I I I}$.

In textured samples, only one group of diffraction peaks is accessible with $\theta-2 \theta$ scans. One can find that the (220) peak intensity of Heusler compounds is independent of site-swap disorder 38 by an analysis of the structure factors of a $\mathrm{C}_{b}$ (half) or $\mathrm{L}_{1}$ (full-)Heusler compound. In contrast, the (200) and (111) peak intensities depend on disorder and will vanish for A1 type disorder, where the lattice sites are randomly occupied. Therefore a (110)-textured L $2{ }_{1}$ film will give the same specular diffractogram as a (110)-textured A1-disordered film of the same compound. Furthermore, this is also true for the various crystal structures which have been proposed for $\mathrm{CoMnSb}$ (see Sec. II).

To overcome this problem, non-specular $\omega-2 \theta$ scans were taken with various settings of $\psi$ and $2 \theta=2 \cdot \omega$. Figure 5 presents a set of these scans taken on sample Half $\mathrm{B}^{I}\left(400^{\circ} \mathrm{C}\right)$. The scan with $\psi=0^{\circ}$ shows the $\mathrm{V}$ (110) reflex and the strong (440) reflex from the Co-MnSb. At higher values of $\psi$ more reflections appear. A well-ordered $\mathrm{C}_{b}$ lattice would exhibit the (222), (400) and (440) reflections only (which would be indexed (111), (200) and (220) then). The additional (311), (331), $(333) /(511)$ and (531) peaks have comparatively low in-

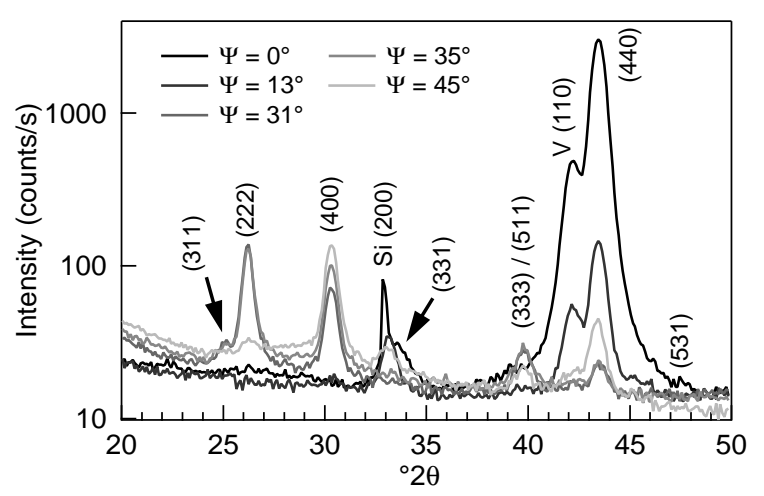

FIG. 5. Diffraction patterns of sample Half $\mathrm{B}^{I}\left(400^{\circ} \mathrm{C}\right)$ taken under various tilt angles $\Psi$. The peaks not labeled by V or Si correspond to Co-Mn-Sb.

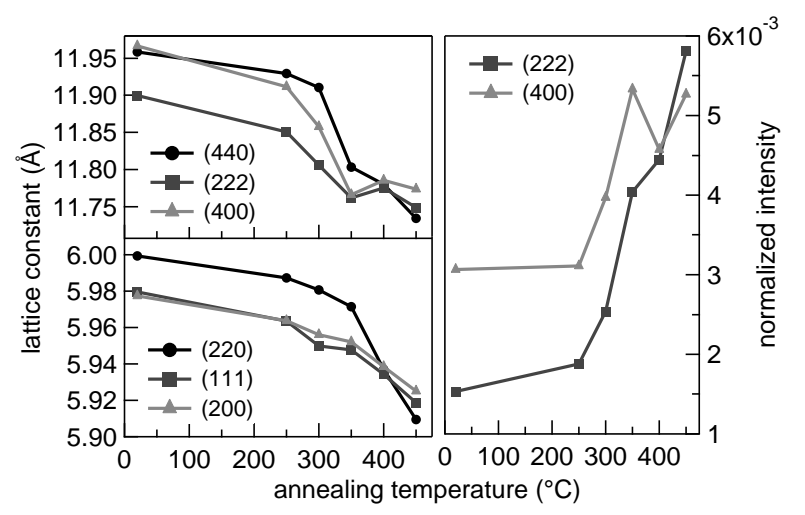

FIG. 6. Left: Lattice constants of sample Half $\mathrm{B}^{I}$ (top) and sample Half $\mathrm{B}^{I I I}$ (bottom), determined on three independent reflexes at the corresponding tilt angles. Right: Normalized integrated intensity of the (222) and (400) peaks for sample Half $\mathrm{B}^{I}$. (220)/(440) reflections were taken at $\Psi=0.00^{\circ}$, $(111) /(222)$ at $\Psi=35.26^{\circ}$ and $(200) /(400)$ at $\Psi=45.00^{\circ}$.

tensity and grow with increasing annealing temperature. Similar patterns were found by Ksenofontov et $a l^{25}$ in powders of $\mathrm{Co}-\mathrm{Mn}-\mathrm{Sb}$. The peaks can not be indexed based on a $\mathrm{L} 2{ }_{1} / \mathrm{C} 1_{b}$ structure with a lattice constant of about $5.9 \AA$. In contrast, they can be indexed by the assumption of a doubled unit cell parameter of $11.77 \AA$.

Analogous measurements on sample Half $\mathrm{B}^{I I I}$ did not show the additional peaks. The complex unit cell with a doubled lattice constant is obviously specific for the nearly stoichiometric CoMnSb. The Co excess in films sputtered from target III might lead to occupation of the vacant sites in the $\mathrm{MnSb}$ substructure of the $\mathrm{Co}_{2} \mathrm{MnSb}$ $\mathrm{MnSb}$ super cells. If finally one Co atom per f.u. is added (without reducing the $\mathrm{Sb}$ concentration at the same time), the system will be in the $L 2_{1}$ structure.

In Figure 6 we determine the in-plane and out-of-plane lattice parameters as a function of the annealing temper- 
ature for sample Half $\mathrm{B}^{I}$ and sample Half $\mathrm{B}^{I I I}$. The lattice parameters shrink with increasing annealing temperature, indicating a lattice relaxation and/or ordering. After $400^{\circ} \mathrm{C}$ the lattice parameters in the three probed directions are the same, stress-induced influences might be compensated.

The integrated intensity of the (222) and (400) reflexes is a measure for the order in the films. Figure 6 (right) shows a plot of the normalized integrated (222) and (400) peak intensities as a function of the annealing temperature. The normalization is done with respect to the integrated (440) intensity to correct for recrystallization. The (222) intensity increases by a factor of 4 from the as-prepared sample to $450^{\circ} \mathrm{C}$. Note that the intensity of the (222) reflex is further increased with annealing from $400^{\circ} \mathrm{C}$ to $450^{\circ} \mathrm{C}$, whereas the (400) reflex intensity does not increase monotonously, but rather shows a dip at $T_{A}=400^{\circ} \mathrm{C}$.

The additional peaks of the CoMnSb superstructure vanish for annealing at $T_{A}=450^{\circ} \mathrm{C}$. Vanadium is implemented into the lattice and thus decreases the saturation magnetization (Section IIIA). The dip in the normalized (400) intensity curve at $400^{\circ} \mathrm{C}$ hints the incipient diffusion of $\mathrm{V}$, changing the properties of the lattice. However, the face-centered cubic structure is preserved and the $\mathrm{V}$ increases the structure factor of the (222) reflex.

\section{THE CO-MN-SB / AL-O INTERFACE}

\section{A. X-ray absorption spectroscopy (XAS)}

The influence of the $\mathrm{Al}$ thickness and the annealing on the chemical states of $\mathrm{Co}$ and $\mathrm{Mn}$ at the $\mathrm{Co}-\mathrm{Mn}-\mathrm{Sb} /$ $\mathrm{Al}-\mathrm{O}$ interface was investigated by surface-sensitive XAS and XMCD in total electron yield (TEY) mode applied to Half $\mathrm{C}^{I I}$ samples, involving a plasma oxidized wedge of $\mathrm{Al}$ on top of Co-Mn-Sb. The Co-Mn-Sb/Al-O interface needs to be optimized with respect to the oxidation process of the Aluminum in order to reach maximum TMR ratios $37,39,40$.

An increasing $\mathrm{Mn}$ to Co concentration ratio was found at the interface with decreasing $\mathrm{Al}$ thickness (see $I_{L 3}^{M n} / I_{L 3}^{C o}$ in Fig. 7 .). This trend becomes more pronounced after annealing. The shapes of the Mn XA spectra in Figure 8 show that this Mn segregation at the $\mathrm{Co}-\mathrm{Mn}-\mathrm{Sb} / \mathrm{Al}-\mathrm{O}$ interface is caused by $\mathrm{MnO}$ formation at the lower barrier interface. For an $\mathrm{Al}$ thickness of only $1.0 \mathrm{~nm}$ the spectra of the as prepared as well as the annealed samples were dominated by the multiplet structure typical for $\mathrm{MnO}^{41}$. The multiplet structure became weaker with increasing $\mathrm{Al}$ thickness. A structureless $L_{2,3}$ absorption edge was found for thick Aluminum, its shape is similar to metallic bulk $\mathrm{Mn}^{41}$.

The significant formation of interfacial $\mathrm{MnO}$ (paramagnetic at room temperature ${ }^{42}$ ) for $1.0 \mathrm{~nm}$ thick $\mathrm{Al}$ is also reflected in the low XMCD asymmetry ratio $\xi^{M n} / \xi^{C o}$. This is proportional to the ratio of the Mn and Co mag-
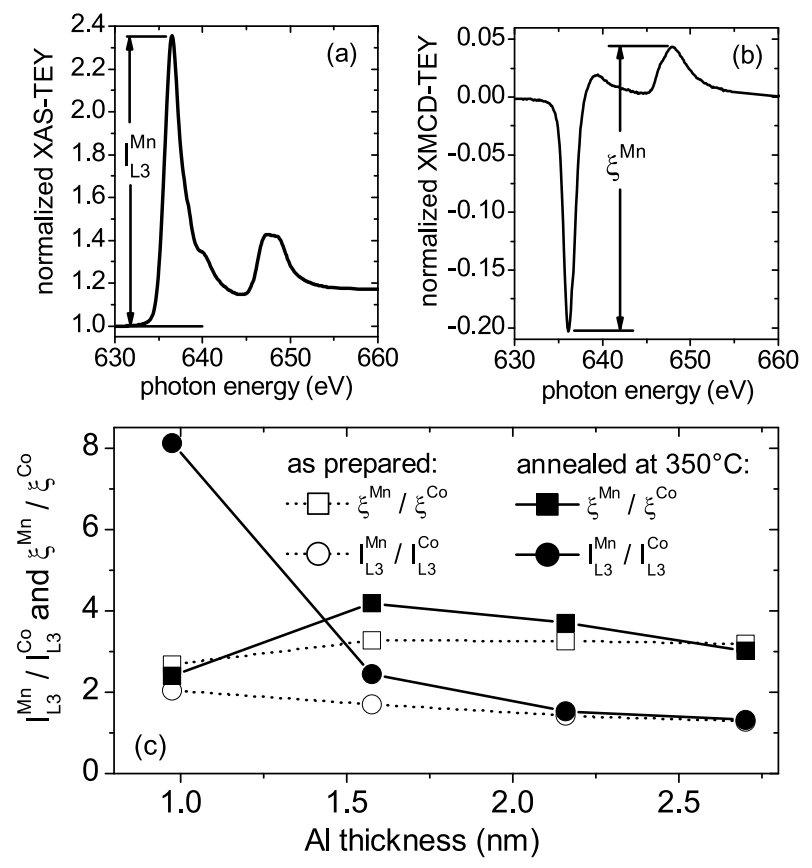

FIG. 7. (a) XA-TEY spectrum at the Mn $L_{2,3}$ edges of sample Half $\mathrm{C}^{I I}$ (Al thickness: $1.6 \mathrm{~nm}$ ) after ex-situ annealing at $350^{\circ} \mathrm{C}$. The maximum intensity of the $L_{3}$ resonance is defined as $I_{L 3}^{M n}$. (b) Corresponding XMCD-TEY spectrum. The definition of the maximum XMCD asymmetry $\xi^{M n}$ is shown. (c) Summary of the intensity and asymmetry ratios $I_{L 3}^{M n} / I_{L 3}^{C o}$ and $\xi^{M n} / \xi^{C o}$ of sample Half $\mathrm{C}^{I I}$.

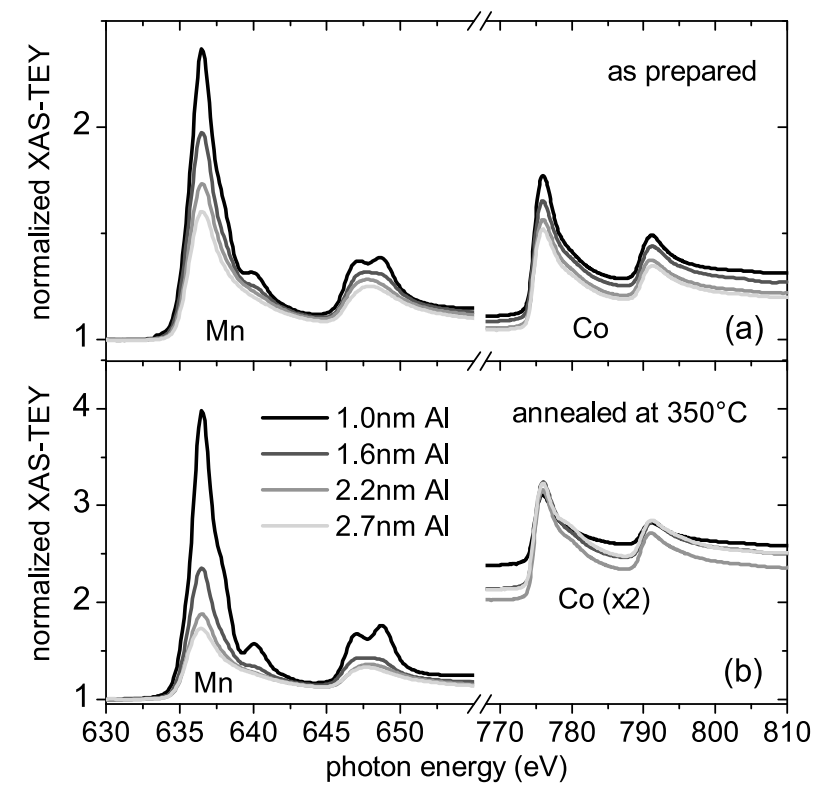

FIG. 8. XA-TEY spectra at the Co and $\mathrm{Mn} L_{2,3}$ edges of sample Half $\mathrm{C}^{I I}$. 


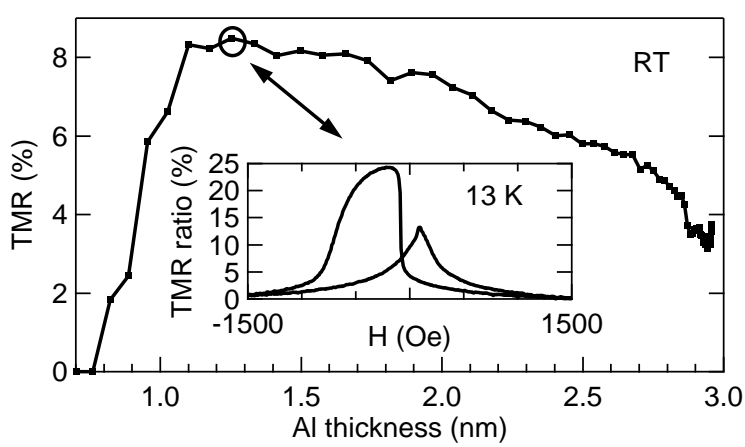

FIG. 9. TMR as a function of the Al thickness of Full ${ }^{I I}$. The measurements were taken with a dc bias voltage of $10 \mathrm{mV}$.

netic moments, which are both dominated by the spin moment (see above). $\xi^{M n} / \xi^{C o}$ was maximal after annealing for an $\mathrm{Al}$ thickness of $1.6 \mathrm{~nm}$ and the contribution of $\mathrm{MnO}$ to the XA spectrum is already very small compared to $1.0 \mathrm{~nm} \mathrm{Al}$ (see Figure 8 $\mathrm{b}$ ). Here, the best atomic order was achieved, since disorder is expected to reduce the $\mathrm{Mn}$ to Co magnetic moment ratio (see Sec. IIIC). While thin $\mathrm{Al}$ leads to an oxidation of the ferromagnetic electrode, metallic $\mathrm{Al}$ will remain at the interface, if the $\mathrm{Al}$ thickness is too large. This $\mathrm{Al}$ can diffuse into the $\mathrm{Co}-\mathrm{Mn}-\mathrm{Sb}$ electrode during annealing leading to a reduction of the interfacial magnetic moments and the $\mathrm{Mn}$ to Co magnetic moment ratio. The observed increase of the $\mathrm{Mn}$ to Co (bulk) spin moment ratio of all films discussed in the previous Sec. $[I \mathrm{C}$ was not found at the Co-Mn-Sb/Al-O interface for $2.7 \mathrm{~nm}$ thick $\mathrm{Al}$ due to residual unoxidized Al. However, such an increase was found for optimum aluminum thickness of $1.6 \mathrm{~nm}$. At this $\mathrm{Al}$ thickness, the properties of the Co-Mn-Sb near the surface are closest to those of the bulk material.

\section{B. Transport properties of magnetic tunnel junctions with Co-Mn-Sb electrode}

The magnetic tunnel junctions Full ${ }^{I I}$ were prepared with optimized growth conditions as discussed earlier: they were in-situ annealed at $350^{\circ} \mathrm{C}$ after $150 \mathrm{~s}$ plasmaoxidation of the $\mathrm{Al}$ wedge. In order to remove contaminations from the surface, the samples were plasma oxidized for another $50 \mathrm{~s}$ after the annealing. Subsequently, ex-situ vacuum annealing at $275^{\circ} \mathrm{C}$ in a magnetic field of $0.1 \mathrm{~T}$ was employed to set the exchange bias of the pinned electrode. In previous papers $\frac{13,43}{3}$ we show that this preparation scheme is suitable to produce magnetic tunnel junctions with Heusler compound electrodes that exhibit high TMR ratios. Patterning was done by optical lithography and ion beam etching, the transport properties of the quadratic $100 \times 100 \mu \mathrm{m}^{2}$ wide MTJs were measured by conventional two-probe method with a constant dc bias voltage.
In Figure 9 the dependence of the TMR on the $\mathrm{Al}$ thickness is shown. A maximal TMR ratio of $8.5 \%$ at room temperature was found at an $\mathrm{Al}$ thickness of 1.25 $\mathrm{nm}(1.6 \mathrm{~nm}$ AlOx). The Al thickness dependence of $\xi^{M n} / \xi^{C o}$ presented in the last section fits quite well to the Al thickness dependence of the TMR. The observed maximum TMR ratio at $13 \mathrm{~K}$ was $24 \%$ (see inset in Figure 9). According to the Jullière model, the effective tunneling spin polarization is calculated to be 0.22 , using a spin polarization for $\mathrm{Co}_{70} \mathrm{Fe}_{30}$ of $0.5 \underline{\underline{44}}$. No higher values for the TMR ratio could be found with varied film growth parameters. Moreover, MTJs with Co-Mn-Sb electrodes from targets I and III show comparable TMR ratios. Taking this together with the careful preparation of the films and the tunnel barrier, we conclude that the low observed spin polarization is an intrinsic property of our $\mathrm{Co}-\mathrm{Mn}-\mathrm{Sb}$ electrodes.

\section{v. CONCLUSION}

It was shown that post annealing increases the atomic order and the magnetization of the Co-Mn-Sb films, while the Co to $\mathrm{Mn}$ magnetic moment ratio decreased. The samples degraded for temperatures higher than $350^{\circ} \mathrm{C}$ because of inter-diffusion of $\mathrm{V}$ at the $\mathrm{Co}-\mathrm{Mn}-\mathrm{Sb} / \mathrm{V}$ interface. Adding Co to the samples leads to higher magnetization values and to a larger Co to Mn magnetic moment ratio as expected from simple arguments basing on the typical nearest-neighbor coupling behavior of $\mathrm{Co}-\mathrm{Co}$ and $\mathrm{Mn}-\mathrm{Mn}$ pairs. A parallel coupling of the $\mathrm{Co}$ and Mn magnetic spin moments was found for all compositions. For nearly stoichiometric films the Co to Mn spin moment ratio was $+18.8 \%$ after annealing and the unit cell parameter was $11.77 \AA$. This fits the expectations for CoMnSb crystallized in a complex $\mathrm{Co}_{2} \mathrm{MnSb} / \mathrm{MnSb}$ super structure as reported by Ksenofontov et al $\stackrel{25}{\underline{ } \text {. The }}$ addition of $\mathrm{Co}$ as well as interdiffusion from the vanadium buffer destroy the super structure. Finally, the transport properties of magnetic tunnel junctions with $\mathrm{Co}-\mathrm{Mn}-\mathrm{Sb}$ electrode, $\mathrm{Al}-\mathrm{O}$ barrier and $\mathrm{Co}-\mathrm{Fe}$ counter electrode are in agreement with the predicted low spin polarization: we found an effective tunneling spin polarization of only $22 \%$, which clearly indicates a non-half metallic character of $\mathrm{CoMnSb}$.

\section{ACKNOWLEDGEMENT}

The authors gratefully acknowledge financial support by the Deutsche Forschungsgemeinschaft (DFG) and the Deutscher Akademischer Austausch Dienst (DAAD) and the opportunity to work at BL 6.3.1 of the Advanced Light Source, Berkeley, USA. The ALS is supported by the U.S. Department of Energy under Contract No. DEAC02-05CH11231. For financial support of the XRD measurements under contract No.DAAD/10/2006 M.M., 
T.S. and J.K. gratefully acknowledge the Ministry of Science and Higher Education of Poland.

${ }^{1}$ S. A. Wolf, D. D. Awschalom, R. A. Buhrman, J. M. Daughton, S. von Molnar, M. L. Roukes, A. Y. Chtchelkanova, and D. M. Treger, Science 294, 1488 (2001).

${ }^{2}$ G. Reiss and D. Meyners, Appl. Phys. Lett. 88, 043505 (2006).

${ }^{3}$ A. Thomas, D. Meyners, D. Ebke, N. Liu, M. D. Sacher, J. Schmalhorst, G. Reiss, H. Ebert, and A. Hütten, Appl. Phys. Lett. 89, 012502 (2006).

${ }^{4}$ M. Julliere, Phys. Lett. 54A, 225 (1975).

${ }^{5}$ R.A. de Groot, F.M. Mueller, P.G. vanEngen, and K.H.J. Buschow, Phys. Rev. Lett. 50, 2024 (1983).

${ }^{6}$ S. Ishida, T. Masaki, S. Fujii, and S. Asano, Physica B 245, 1 (1998).

${ }^{7}$ I. Galanakis, P. H. Dederichs, and N. Papanikolaou, Phys. Rev. B 66, 174429 (2002).

${ }^{8}$ S. Wurmehl, G.H. Fecher, H. C. Kandpal, V. Ksenofontov, C. Felser, H.-J. Lin, and J. Morais, Phys. Rev. B 72, 184434 (2005).

${ }^{9}$ Half-metallic Alloys: Fundamentals and Application, I. Galanakis and P. H. Dedrichs (Eds.), Lecture Notes in Physics 676, Springer Berlin Heidelberg (2005), ISBN-10 3-540-27719-6.

${ }^{10}$ K. Inomata, N. Tezuka, S. Okamura, H. Kobayashi, and A. Hirohata, J. Appl. Phys. 95, 7234 (2004).

${ }^{11}$ J. Schmalhorst, S. Kämmerer, G. Reiss, and A. Hütten, Appl. Phys. Lett. 86, 052501 (2005).

${ }^{12}$ M. Oogane, Y. Sakuraba, J. Nakata, H. Kubota, Y. Ando, A. Sakuma, and T. Miyazaki, J. Phys. D: Appl. Phys. 39, 834 (2006).

${ }^{13}$ D. Ebke, J. Schmalhorst, N.-N. Liu, A. Thomas, G. Reiss, and A. Hütten, Appl. Phys. Lett. 89, 162506 (2006).

${ }^{14}$ Y. Sakuraba, M. Hattori, M. Oogane, Y. Ando, H. Kato, A. Sakuma, T. Miyazaki, and H. Kubota, Appl. Phys. Lett. 88, 192508 (2006).

${ }^{15}$ J. Schmalhorst, A. Thomas, S. Kämmerer, O. Schebaum, D. Ebke, M. D. Sacher, G. Reiss, A. Hütten, A. Turchanin, A. Gölzhäuser, et al., Phys. Rev. B 75, 014403 (2007).

${ }^{16}$ J. Schmalhorst, D. Ebke, A. Weddemann, A. Hütten, A. Thomas, G. Reiss, A. Turchanin, A. Gölzhäuser, B. Balke, C. Felser, J. Appl. Phys. 104, 043918 (2008).

${ }^{17}$ C. T. Tanaka, J. Nowak, and J. S. Moodera, J. Appl. Phys. 86, 6239 (1999)

${ }^{18}$ T. Ishikawa, S. Hakamata, K. Matsuda, T. Uemura, and M. Yamamoto, J. Appl. Phys. 103, 07A919 (2008).

${ }^{19}$ S. Ikeda, J. Hayakawa, Y. Ashizawa, Y. M. Lee, K. Miura, H. Hasegawa, M. Tsunoda, F. Matsukura, and H. Ohno, Appl. Phys. Lett. 93, 082508 (2008).

${ }^{20}$ I. Galanakis, J. Phys.: Condens. Matter 14, 6329 (2002).
${ }^{21}$ M. J. Otto, H. Feil, R. A. M. van Woerden, J. Wijngaard, P. J. van der Valk, C. F. van Bruggen, and C. Haas, J. Magn. Magn. Mat. 70, 33 (1987).

${ }^{22}$ J. Tobola and J. Pierre, J. Alloys and Compd. 296, 243 (2000).

${ }^{23}$ J. P. Senateur, A. Rouault, R. Fruchart, and D. Fruchart, J. Solid State Chem. 5, 226 (1972).

${ }^{24}$ K. Kaczmarska, J. Pierre, J. Tobola, and R. V. Skolozdra, Phys. Rev. B 60, 373 (1999).

${ }^{25}$ V. Ksenofontov, G. Melnyk, M. Wojcik, S. Wurmehl, K. Kroth, S. Reiman, P. Blaha, and C. Felser, Phys. Rev. B 74, 134426 (2006).

${ }^{26}$ J. Schmalhorst, H. Brückl, G. Reiss, G. Gieres, and J. Wecker, Journal of Appl. Physics 91, 6617 (2002).

${ }^{27}$ D. Briggs and M. P. Seah, Practical Surface Analysis, Chapter 4: Depth profiling in AES and XPS (John Wiley \& Sons, 1990).

${ }^{28}$ The depth resolution of the profiles can be calculated from the slope of the $\mathrm{V}$ intensity at the $\mathrm{V} / \mathrm{SiO}_{2}$ interface 27 . The slope is very similar for the as prepared and the $450^{\circ} \mathrm{C}$ samples, the sputter time needed to reduce the intensity from $84 \%$ to $16 \%$ of the maximum $\mathrm{V}$ intensity is $20.7 \mathrm{~min}$ (as prepared) and $20.4 \mathrm{~min}$ $\left(450^{\circ} \mathrm{C}\right)$, respectively. By comparing these sputter times with the required $214 \mathrm{~min}$ to sputter through the $40 \mathrm{~nm}$ thick V layer of the as prepared sample an identical depth resolution of about $4 \mathrm{~nm}$ can be estimated for all three samples.

${ }^{29}$ Y. U. Idzerda, C. T. Chen, H.-J. Lin, G. Meigs, G. H. Ho, and C.C. Kao, Nucl. Instrum. Methods Phys. Res. A 347, 134 (1994).

${ }^{30}$ C. T. Chen, Y. U. Idzerda, H.-J. Lin, N. V. Smith, G. Meigs, E. Chaban, G. H. Ho, E. Pellegrin, and F. Sette, Phys. Rev. Lett. 75, 152 (1995).

${ }^{31}$ J. Stöhr, J. Elect. Spect. Rel. Phen. 75, 253 (1995).

${ }^{32}$ J. Schmalhorst, D. Ebke, M. Meinert, A. Thomas, G. Reiss, and E. Arenholz, J. Appl. Phys. 105, 053906 (2009).

${ }^{33}$ J. Schmalhorst, S. Kämmerer, M. Sacher, G. Reiss, A. Hütten, and A. Scholl, Phys. Rev. B 70, 024426 (2004).

${ }^{34}$ N. D. Telling, P. Keatley, G. van der Laan, R. J. Hicken, E. Arenholz, Y. Sakuraba, M. Oogane, Y. Ando, K. Takanashi, A. Sakuma, et al., Phys. Rev. B 78, 184438 (2008).

${ }^{35}$ I. Galanakis, Phys. Rev. B 71, 012413 (2005).

${ }^{36}$ S. Picozzi, A. Continenza, and A. J. Freeman, Phys. Rev. B 69, 094423 (2004).

${ }^{37}$ J. Schmalhorst, D. Ebke, M. D. Sacher, N.-N. Liu, A. Thomas, G. Reiss, and A. H. E. Arenholz, IEEE Trans. Magn. 64, 2806 (2007).

${ }^{38}$ P. Webster, Contemp. Phys. 10, 559 (1969).

${ }^{39}$ J. Schmalhorst, M. D. Sacher, A. Thomas, H. Brückl, G. Reiss, and K. Starke, J. Appl. Phys. 97, 123711 (2005).

${ }^{40}$ M. D. Sacher, PhD Thesis, Bielefeld University, Germany (2008).

${ }^{41}$ Y. Yonamoto, T. Yokoyama, K. Amemiya, D. Matsumura, and T. Ohta, Phys. Rev. B 63, 214406 (2001).

${ }^{42}$ G. Pepy, J. Phys. Chem. Solids 35, 433 (1974).

${ }^{43}$ S. Kämmerer, A. Thomas, A. Hütten, G. Reiss, Appl. Phys. Lett. 85, 79 (2004).

${ }^{44}$ A. Thomas, Ph.D. thesis, University of Bielefeld (2003). 